\title{
新型罗丹明类 $\mathrm{Ca}^{2+}$ 荧光探针的合成及光谱性能研究
}

\author{
苏文崎童 杰杨秉勤* \\ (西北大学化学与材料科学学院 合成与天然功能分子化学教育部重点实验室 西安 710069)
}

\begin{abstract}
摘要 以 2-氨基酚和 3-(二乙基氨基)苯酚为原料, 经过 5 步反应合成了新型罗丹明类荧光探针化合物, 研究发现该化合 物对 $\mathrm{Ca}^{2+}$ 具有较高的选择识别性能, 并且受常见离子的干扰较小. 探针的荧光强度与 $\mathrm{Ca}^{2+}$ 浓度 $\left(2 \times 10^{-6} \sim 5 \times 10^{-5}\right.$ $\mathrm{mol} / \mathrm{L}$ )呈较好的线性关系.
\end{abstract}

关键词 荧光探针; 罗丹明; 钙离子; 识别; 荧光强度

\section{Synthesis and Characterization of A New Rhodamine Fluorescence Molecular Chemosensor for Calcium Ion}

\author{
$\mathrm{Su}$, Wenqi Tong, Jie Yang, Bingqin* \\ (Key Laboratory of Synthetic and Natural Functional Molecule Chemistry of Ministry of Education, Department of Chemistry, \\ Northwest University, Xi'an 710069)
}

\begin{abstract}
The novel rhodamine fluorescent chemosensor was synthesized in five steps from 2-aminophenol and 3-(diethylamino)phenol. It exhibited very strong fluorescence responses to $\mathrm{Ca}^{2+}$ and had remarkably high selectivity to $\mathrm{Ca}^{2+}$ than other metal ions. When the concentration of $\mathrm{Ca}^{2+}$ was in the range of $2 \times 10^{-6} \sim 5 \times 10^{-5} \mathrm{~mol} / \mathrm{L}$, there was a good linearity between the fluorescence intensity of the chemosensor and the concentration of $\mathrm{Ca}^{2+}$.

Keywords fluorescent chemosensor; rhodamine; calcium ion; recognition; fluorescence intensity
\end{abstract}

钙是机体各项生理活动不可缺少的元素，对于维持 细胞膜两侧的生物电位，能维持正常的神经传导功能、 正常的肌肉伸缩与舒张功能、神经一肌肉传导功能 ${ }^{[1 \sim 4]}$. 一旦生物体内钲元素缺乏, 就会发生免疫系统功能下 降, 引发疾病. 精确检测细胞内钻元素含量及其转化已 成为化学界、生物界及医学界的重要研究课题 ${ }^{[\sim 9]}$.

近年来, 人们开始不断研究生物体细胞内 $\mathrm{Ca}^{2+}$ 的 检测技术和方法, 建立和发展了䥻离子传感器、 $\mathrm{Ca}^{2+}$ 荧 光探针和核磁共振等多种方法 ${ }^{[10,11]}$. $\mathrm{Ca}^{2+}$ 苂光探针法通 常具有选择性好、灵敏度高、简便快捷等特点, 因而得 到快速发展, 是目前最常用的一种方法. 尽管许多文 献[12 14]报道了钙离子荧光探针, 但是它们的激发波 长都在紫外区, 检测过程对活体细胞的损伤较大, 同时 也不能避免来自生物体本身自发荧光的影响, 因而并非 理想的苂光探针. 较新报道的苂光探针分子在一定程度 上改善了紫外光对活体细胞的损伤问题 $\left(\lambda_{\mathrm{ex}}>400 \mathrm{~nm}\right)$, 使活体细胞内的钙离子探测成为可能 ${ }^{[15 ~ 18]}$.
罗丹明类苂光染料由于具有摩尔消光系数高、光稳 定性好、苂光量子产率高、较长的激发波长和发射波长 等优异的光物理和光化学性能, 现已成为制备苂光探针

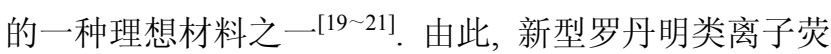
光探针的合成及性质研究吸引了很多科学家的注意. 从 罗丹明类荧光探针对金属离子的识别机理来讲，目前的 文献报道主要是基于罗丹明类化合物螺酰胺环的

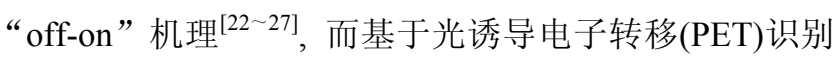
机理的罗丹明类荧光探针还鲜有文献报道. 基于以上思 路, 本文以罗丹明为发色基团, 选取水溶性好、识别性 能高的 APTRA (2-氨基苯氧基- $N, N, O$-三乙酸)作为识别 基团, 利用光诱导电子转移原理(PET), 设计合成了新 型罗丹明类苂光探针化合物 R1, 考察了 R1 在 Tris- $\mathrm{HCl}$ 缓冲溶液体系中加入 $\mathrm{Ca}^{2+}$ 的荧光性能. 发现探针化合 物 $\mathbf{R} 1$ 不仅能准确地识别 $\mathrm{Ca}^{2+}$, 而且受常见金属阳离子 的干扰较小. 为避免活体细胞的损伤, 降低生物体自身 的荧光背景, 进一步改善 $\mathrm{Ca}^{2+}$ 的细胞检测提供了可能.

* E-mail: yangbq@nwu.edu.cn

Received December 4, 2012; revised January 25, 2013; published online February 5, 2013

Project supported by the National Natural Science Foundation of China (No. 21172178).

国家自然科学基金(No. 21172178)资助项目. 
目标化合物 R1 合成路线如 Scheme 1.

\section{1 结果与讨论}

\section{1 合成讨论}

罗丹明类化合物的传统合成方法是通过 3-(烷基氨 基)苯酚与过量的邻苯二甲酸䣶熔化缩合反应 ${ }^{[28]}$ 制得, 反应条件苛刻且生成物产率低. 为了提高产率, 通常增 大 3-(烷基氨基)苯酚的用量, 但反应的后处理比较繁琐, 排放的废弃物对环境会造成严重污染. 有文献[29]报道 罗丹明类染料的新合成方法: 以丙酸作溶剂, 加入催化 量的对甲苯磺酸, 通过 3-(烷基氨基)苯酚与苯甲醛类化 合物在较低温度下反应得到目标化合物, 该方法具有反 应时间较短, 反应温度较低, 且产率有较大提高, 避免 了使用高沸点有机溶剂, 后处理简单等较多优点. 本文 采用类似合成方法，优化反应条件，通过 3-(二乙基氨 基)苯酚与 3-乙氧羰甲氧基-4- $N, N$-二(乙氧羰甲基)氨基 苯甲醛反应得到目标探针化合物 R1.

\section{2 缩合反应中反应物配比的影响}

为了优化反应条件, 在其它反应条件不变的情况 下, 考察了 3-(二乙基氨基)苯酚与 3-乙氧羰甲氧基4- $N, N$-二(乙氧羰甲基)氨基苯甲醛的物质的量比对最终 反应产率的影响. 由表 1 可以看出, 3-(二乙基氨基)苯酚 的用量应稍大于剂量比, 这样有利于反应产率升高, 而 当反应物比率增大到 $3: 1$ 时, 反应产率反而有所下降. 通过实验，确定了最佳反应物质的量比为 $2.5: 1$, 此时

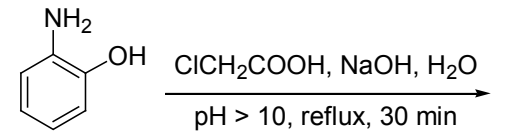

1

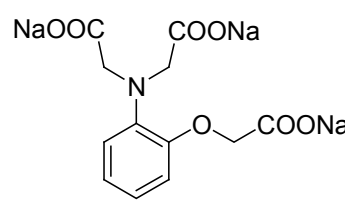

2
反应产率最高.

表 1 反应物的物质的量比对产率的影响

Table 1 Effect of molar ratio of raw materials on the yield

\begin{tabular}{lccccc}
\hline 反应物的物质的量比 & $2.0: 1$ & $2.2: 1$ & $2.3: 1$ & $2.5: 1$ & $3.0: 1$ \\
\hline 产率 $\%$ & 16.4 & 20.3 & 21.7 & 25.0 & 23.1 \\
\hline
\end{tabular}

\section{3 化合物 R1 的光谱性能研究}

\subsection{1 化合物 $\mathbf{R} 1$ 对金属离子的选择性识别}

为了确定化合物 R1 对金属离子的识别性能，选择 碱金属及碱土金属 $\left(\mathrm{Na}^{+}, \mathrm{K}^{+}, \mathrm{Mg}^{2+}, \mathrm{Ca}^{2+}\right)$ 和过渡金属 $\left(\mathrm{Zn}^{2+}, \mathrm{Cd}^{2+}, \mathrm{Co}^{2+}, \mathrm{Ni}^{2+}, \mathrm{Cu}^{2+}, \mathrm{Mn}^{2+}, \mathrm{Ag}^{+}, \mathrm{Hg}^{2+}, \mathrm{Cr}^{3+}\right.$, $\mathrm{Fe}^{3+}$ )及 $\mathrm{Al}^{3+}, \mathrm{Sn}^{2+}$ 为识别对象(图 1), 在 $0.01 \mathrm{~mol} / \mathrm{L}$ Tris- $\mathrm{HCl}$ 缓冲溶液体系中(包括 $100 \mathrm{mmol} \cdot \mathrm{L}^{-1} \mathrm{KCl}, \mathrm{pH}=$ 7.2 , 激发波长为 $520 \mathrm{~nm}$ )进行荧光测定. 实验发现，未 加 $\mathrm{Ca}^{2+}$ 之前, 化合物 $\mathbf{R} 1$ 本身苂光强度较弱, 而 $\mathrm{Ca}^{2+}$ 加 入后荧光强度明显增强，这是由于探针化合物是基于 PET 机理导致荧光强度发生猝灭, 即由于识别基团通过 间隔基(苯环)与发色团(罗丹明)相连时, $\mathrm{N}$ 原子上的电子 跃迁至由于受激发而空出的罗丹明母体的基态轨道, 从 而造成激发电子无法回到基态而导致的苂光猝灭，当加 入 $\mathrm{Ca}^{2+}$ 后，化合物 $\mathbf{R} 1$ 和 $\mathrm{Ca}^{2+}$ 络合，阻碍了邻氨基酚衍 生物上原子的电子向罗丹明荧光团的转移, 引起探针化 合物 $\mathbf{R} 1$ 苂光明显增强, 且稳定性很好, 最大发射波长 在 $576 \mathrm{~nm}$ 处, 而同样条件下金属离子 $\mathrm{Na}^{+}, \mathrm{K}^{+}, \mathrm{Zn}^{2+}$, $\mathrm{Cd}^{2+}, \mathrm{Co}^{2+}, \mathrm{Ni}^{2+}, \mathrm{Cu}^{2+}, \mathrm{Ag}^{+}, \mathrm{Hg}^{2+}, \mathrm{Cr}^{3+}, \mathrm{Fe}^{3+}, \mathrm{Mn}^{2+}$,

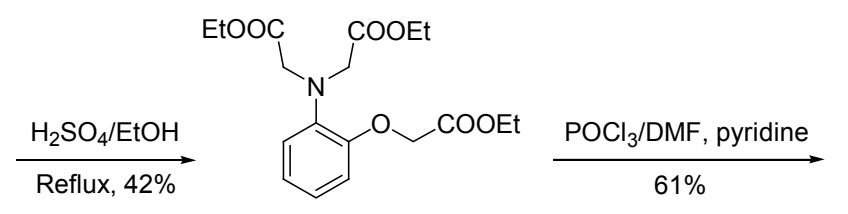<smiles></smiles><smiles>CCOC(=O)COc1cc(C=O)ccc1N(CC(=O)OCC)CC(=O)OCC</smiles>

(1) 3-Diethylaminophenol methylacetic/p-TsOH overnight, $65{ }^{\circ} \mathrm{C}$

(2) Chloranil, $\mathrm{CH}_{2} \mathrm{Cl}_{2} / \mathrm{MeOH}$

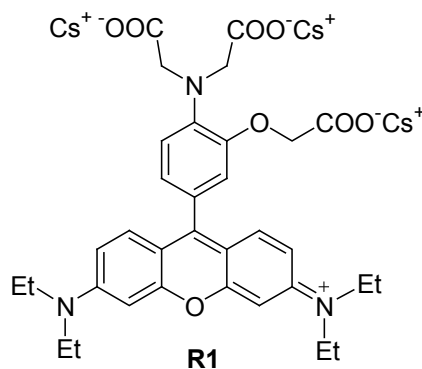

Scheme 1 
$\mathrm{Al}^{3+}, \mathrm{Sn}^{2+}$ 却不能引起苂光强度的变化, 碱土金属离子 $\mathrm{Mg}^{2+}$ 的菼光强度有小幅增加, 但其增加值显著小于探 针对 $\mathrm{Ca}^{2+}$ 的响应值. 说明 $\mathbf{R} 1$ 是一个较高选择性荧光增 强型 $\mathrm{Ca}^{2+}$ 探针.
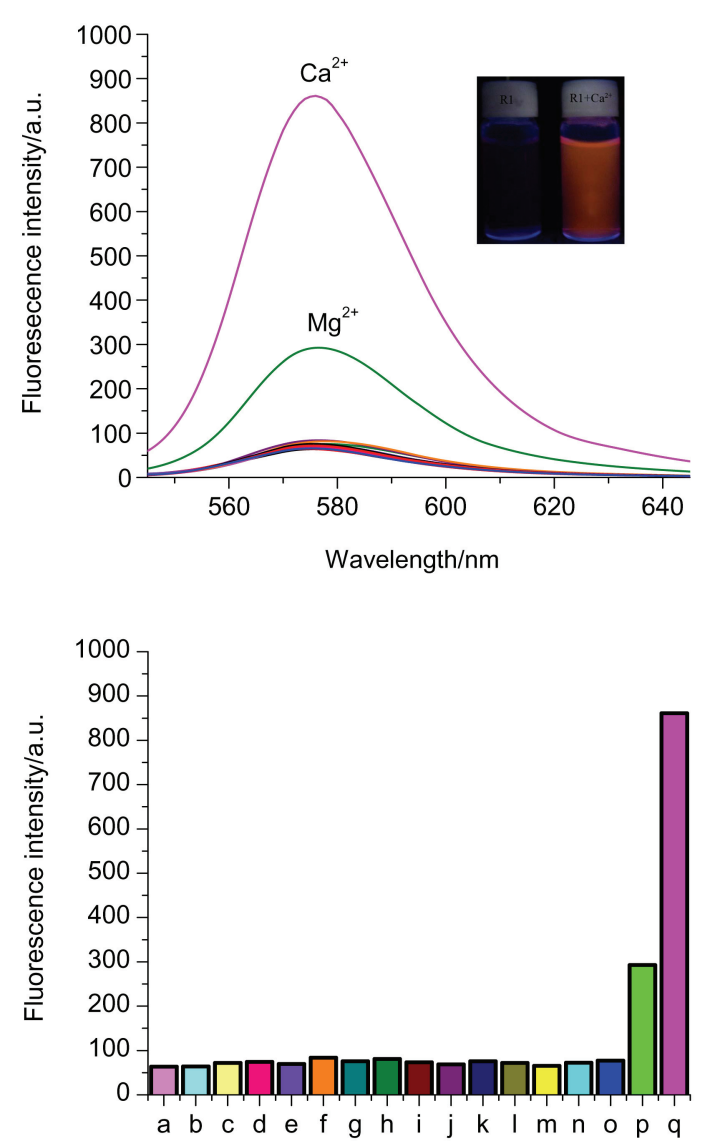

图 $1 \mathrm{R} 1\left(10 \mu \mathrm{mol} \cdot \mathrm{L}^{-1}\right)$ 的金属离子选择性

Figure 1 Fluorescence response of sensor $\mathbf{R} 1\left(10 \mu \mathrm{mol} \cdot \mathrm{L}^{-1}\right)$ to different detected metal ions

(a) compound $\mathbf{R 1}$, (b) $\mathrm{Na}^{+}$, (c) $\mathrm{K}^{+}$, (d) $\mathrm{Cd}^{2+}$, (e) $\mathrm{Mn}^{2+}$, (f) $\mathrm{Ni}^{2+}$, (g) $\mathrm{Co}^{2+}$, (h) $\mathrm{Zn}^{2+}$, (i) $\mathrm{Cu}^{2+}$, (j) $\mathrm{Cr}^{3+}$, (k) $\mathrm{Fe}^{3+}$, (l) $\mathrm{Ag}^{+}$, (m) $\mathrm{Al}^{3+}$, (n) $\mathrm{Sn}^{2+}$, (o) $\mathrm{Hg}^{2+}$, (p) $\mathrm{Mg}^{2+}$, (q) $\mathrm{Ca}^{2+}$

\subsection{3 不同浓度 $\mathrm{Ca}^{2+}$ 对化合物 $\mathbf{R} 1$ 荧光光谱的影响}

在 $0.01 \mathrm{~mol} / \mathrm{L}$ Tris- $\mathrm{HCl}$ 缓冲溶液体系中(包括 100 $\mathrm{mmol} \cdot \mathrm{L}^{-1} \mathrm{KCl}, \mathrm{pH}=7.2$, 激发波长为 $520 \mathrm{~nm}$ ), 测定了 在不同 $\mathrm{Ca}^{2+}$ 浓度条件下化合物 $\mathbf{R} 1$ 溶液的菼光发射光谱 (图 2). 苂光光谱显示, 化合物 $\mathbf{R} 1$ 最大发射波长 $576 \mathrm{~nm}$ 处. 随着 $\mathrm{Ca}^{2+}$ 浓度的逐渐增大, 其最大荧光强度不断增 强，表明 $\mathbf{R} 1$ 与 $\mathrm{Ca}^{2+}$ 存在着良好的络合作用.

化合物 $\mathbf{R} 1$ 对 $\mathrm{Ca}^{2+}$ 响应灵敏. 实验结果显示, $\mathrm{Ca}^{2+}$ 在 $2 \times 10^{-6} \sim 5 \times 10^{-5} \mathrm{~mol} / \mathrm{L}$ 浓度范围内, 随着 $\mathrm{Ca}^{2+}$ 浓度 的逐渐变化, 化合物 $\mathbf{R 1}$ 荧光强度的增强与 $\mathrm{Ca}^{2+}$ 浓度呈 现良好的线性关系(线性相关系数: $R^{2}=0.9926$ )如图 3 所 示. 这表明化合物 $\mathbf{R} 1$ 可以用来定量检测钙离子浓度.

1.3.4 $\mathrm{pH}$ 对化合物 $\mathbf{R} 1$ 和 $\mathbf{R} 1+\mathrm{Ca}^{2+}$ 荧光光谱的影响 $\mathbf{R} 1$ 作为 $\mathrm{Ca}^{2+}$ 的选择性苂光探针主要基于 APTRA
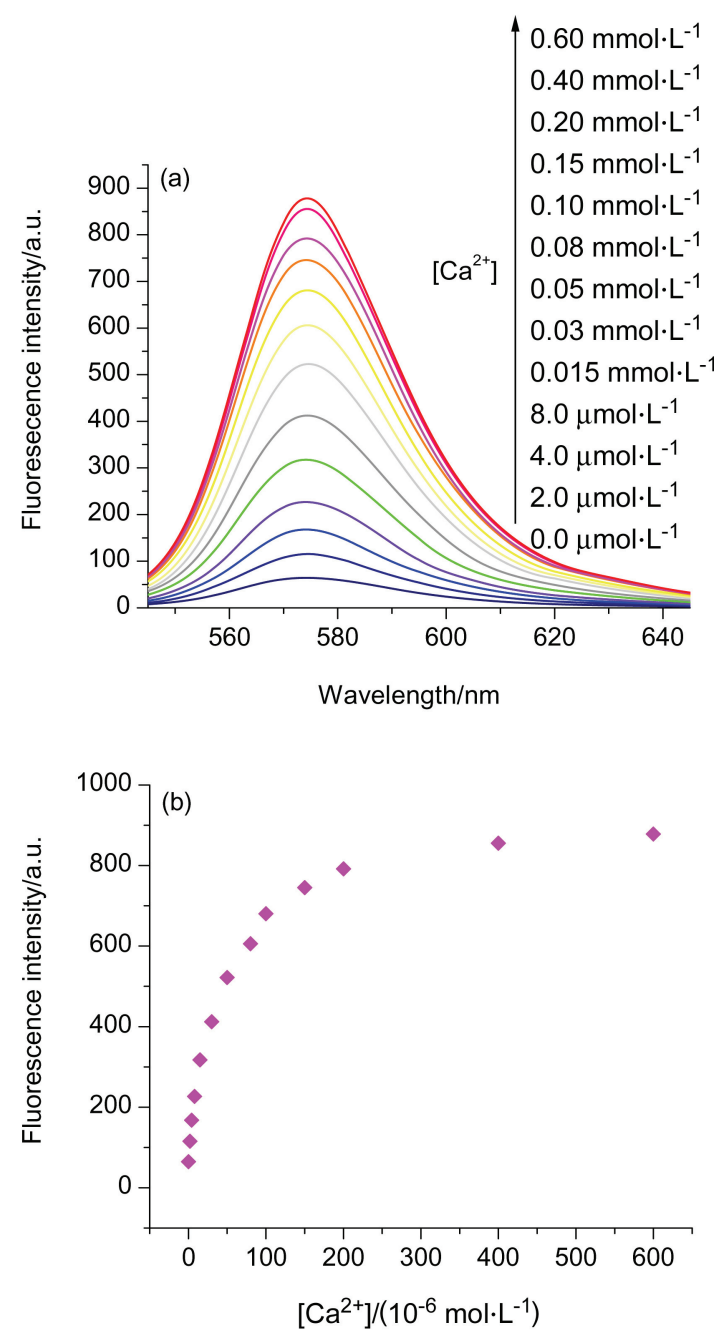

图 2 (a) $\mathbf{R} 1\left(10 \mu \mathrm{mol} \cdot \mathrm{L}^{-1}\right)$ 中逐渐加入 $\mathrm{Ca}^{2+}$ 的荧光发射光谱的 变化曲线图; (b) $\mathbf{R} 1$ 的荧光发射光谱与 $\mathrm{Ca}^{2+}$ 浓度关系图 (激发 波长: $520 \mathrm{~nm}$, 发射波长: $576 \mathrm{~nm}$ )

Figure 2 (a) Fluorescence emission spectra of R1 (10 $\mu \mathrm{mol} \cdot$ $\mathrm{L}^{-1}$ ) in the presence of $\mathrm{Ca}^{2+}$ with concentration increased; (b) Emission spectra of compound $\mathrm{R} 1$ as a function of $\mathrm{Ca}^{2+}$ concentration $\left(\lambda_{\mathrm{ex}}=520 \mathrm{~nm}, \lambda_{\mathrm{em}}=576 \mathrm{~nm}\right)$

的配位作用，而溶液 $\mathrm{pH}$ 值的大小会改变 APTRA 的配位 能力, 进而影响 $\mathbf{R} 1$ 的苂光响应. 实验测定了不同 $\mathrm{pH}$ 值 的 Tris- $\mathrm{HCl}$ 缓冲溶液 $(0.01 \mathrm{~mol} / \mathrm{L})$ 对荧光强度的影响. 图 4 为探针化合物 $\mathbf{R} 1$ 及 $\mathbf{R} 1+\mathrm{Ca}^{2+}$ 分别在其最大发射波 长 $(576 \mathrm{~nm})$ 处的荧光强度与 $\mathrm{pH}$ 关系曲线. 可以看出, $\mathrm{pH}$ 为 5 9 之间, $\mathbf{R} 1$ 的菼光强度变化不大，当加了 $\mathrm{Ca}^{2+}$ 之 后，其苂光强度大幅增强，且在弱酸性至弱碱环境中几 乎不受溶液 $\mathrm{pH}$ 的影响. 由此可见, 探针化合物 R1 在中 性 $\mathrm{pH}$ 范围内可以实现对 $\mathrm{Ca}^{2+}$ 的检测, 这将非常有利于 其在细胞及生物体内进一步的应用.

\subsubsection{R1 与 $\mathrm{Ca}^{2+}$ 的络合比}

据文献 $[16,18]$ 报道, APTRA (2-氨基苯氧基- $N, N, O$ 三乙酸)是一种良好的 $\mathrm{Ca}^{2+}$ 识别基团. 本实验结果也显 


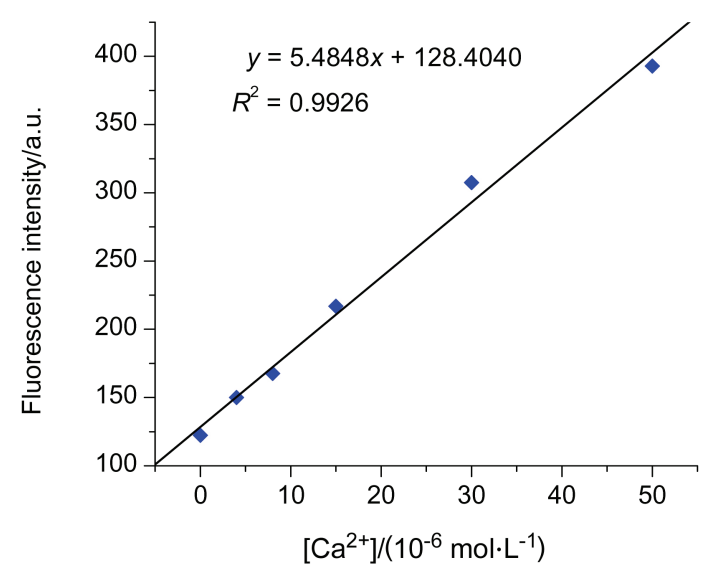

图 $3 \mathbf{R 1}$ 的荧光强度随 $\mathrm{Ca}^{2+}$ 浓度 $\left(2 \times 10^{-6} \sim 5 \times 10^{-5} \mathrm{~mol} / \mathrm{L}\right)$ 变 化的线性关系图

Figure 3 Fluorescence intensity of $\mathbf{R} \mathbf{1}$ as a function of concentration of $\mathrm{Ca}^{2+}\left(2 \times 10^{-6} \sim 5 \times 10^{-5} \mathrm{~mol} / \mathrm{L}\right)$

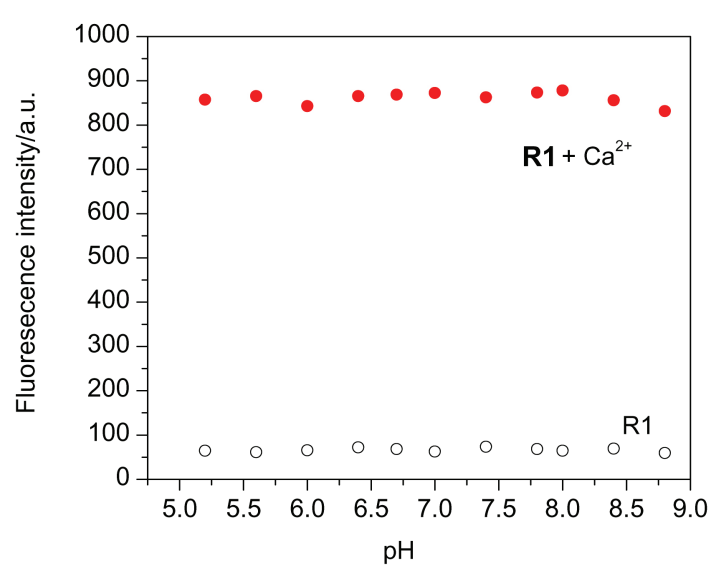

图 $4 \mathrm{pH}$ 对 $\mathbf{R} \mathbf{1}$ 和 $\mathbf{R} 1+\mathrm{Ca}^{2+}$ 苂光强度 $\left(\lambda_{\mathrm{em}}=576 \mathrm{~nm}\right)$ 的影响

Figure 4 Effect of $\mathrm{pH}$ on the emission intensity of $\mathbf{R} \mathbf{1}$ and its $\mathrm{Ca}^{2+}$-complex at $576 \mathrm{~nm}$

示在 $\mathrm{Ca}^{2+}$ 加入后荧光强度明显增强, 说明 APTRA (2-氨 基苯氧基- $N, N, O$-三乙酸)识别基团与 $\mathrm{Ca}^{2+}$ 与探针存在良 好的配位作用, 阻碍或者是减弱了原本化合物 R1 的 PET 过程, 结果使其荧光增强. 为了进一步确定探针化 合物 $\mathbf{R} 1$ 与 $\mathrm{Ca}^{2+}$ 的络合比, 我们做了 $\mathbf{R} 1$ 和 $\mathrm{Ca}^{2+}$ 的络合 曲线. 将 $\mathbf{R} 1$ 和 $\mathrm{Ca}^{2+}$ 的总浓度保持为 $10 \mu \mathrm{mol} / \mathrm{L}$, 改变 $\mathrm{Ca}^{2+}$ 的摩尔分数, 荧光强度在发射波长为 $576 \mathrm{~nm}$ 处随 着 $\mathbf{R} 1$ 和 $\mathrm{Ca}^{2+}$ 的物质的量比变化而变化(图 5). 当 $\mathrm{Ca}^{2+}$ 的摩尔分数为 0.5 时, 探针化合物 $\mathbf{R} 1$ 和 $\mathrm{Ca}^{2+}$ 的络 合物在 $576 \mathrm{~nm}$ 处出现最大的荧光发射, 表明探针化合 物 $\mathbf{R} 1$ 与 $\mathrm{Ca}^{2+}$ 为 $1: 1$ 络合.

\section{2 结论}

综上所述, 本文以罗丹明为发色基团, 以 APTRA (2-氨基苯氧基- $N, N, O$-三乙酸)作为识别基团, 用廉价原

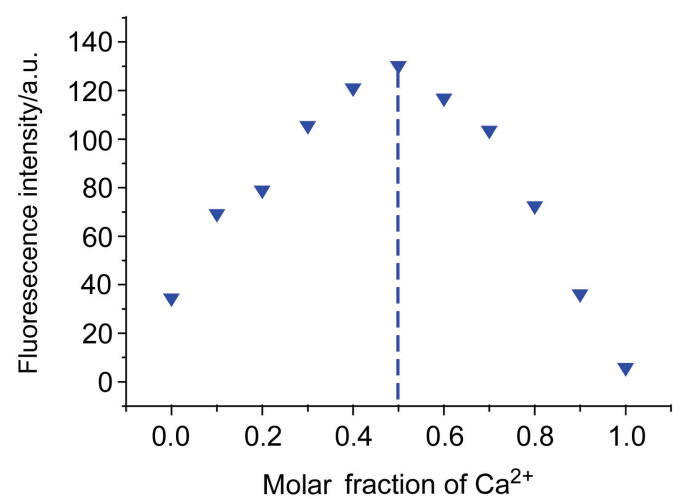

图 $5 \mathrm{R} 1$ 和 $\mathrm{Ca}^{2+}$ 的络合曲线

Figure 5 Job's plot of sensor $\mathbf{R} 1$ and $\mathrm{Ca}^{2+}$

料合成了一种新型罗丹明类 $\mathrm{Ca}^{2+}$ 苂光探针分子 $\mathbf{R} 1$, 并 对所得到的各步产物结构进行了表征. 研究发现, 该探 针分子对 $\mathrm{Ca}^{2+}$ 具有较好的选择识别性, $\mathrm{Ca}^{2+}$ 浓度在 $2 \times 10^{-6} \sim 5 \times 10^{-5} \mathrm{~mol} / \mathrm{L}$ 范围内, 探针的荧光强度与离 子浓度有较好的线性关系, 可以定量检测该范围内 $\mathrm{Ca}^{2+}$ 含量, 对研究该探针化合物在生物体以及环境中的 $\mathrm{Ca}^{2+}$ 识别检测具有一定的指导意义.

\section{3 实验部分}

\section{1 主要仪器与试剂}

WRS-1A 型数字熔点测定仪(上海精密科学仪器有 限公司); Vario EL CHNOS 元素分析仪(德国艾乐曼元素 分析系统公司); INOVA-400 型超导核磁共振仪(TMS 作 内标, 美国 Varian 公司); MALDI-TOF MS 基质辅助飞 行时间质谱仪(Kratos AXIMA-CFR plus Shimadzu Co. UK); F-4500 苂光分光光度计(日本日立公司).

所有试剂均为国产市售分析纯, DMF 和甲醇经干燥 处理, 纯净水 (去离子处理). 金属离子溶液通过 $\mathrm{NaCl}$, $\mathrm{KCl}, \mathrm{Co}\left(\mathrm{NO}_{3}\right)_{2} \cdot 6 \mathrm{H}_{2} \mathrm{O}, \mathrm{Mn}\left(\mathrm{NO}_{3}\right)_{2}, \mathrm{Al}_{2}\left(\mathrm{SO}_{4}\right)_{3}, \mathrm{Zn}\left(\mathrm{NO}_{3}\right)_{2} \bullet$ $6 \mathrm{H}_{2} \mathrm{O}, \mathrm{Ca}\left(\mathrm{NO}_{3}\right)_{2} \cdot 4 \mathrm{H}_{2} \mathrm{O}, \mathrm{Hg}(\mathrm{Cl})_{2}, \mathrm{Cu}\left(\mathrm{NO}_{3}\right)_{2} \cdot 3 \mathrm{H}_{2} \mathrm{O}, \mathrm{SnCl}_{2}$, $\mathrm{Ni}\left(\mathrm{NO}_{3}\right)_{2} \cdot 6 \mathrm{H}_{2} \mathrm{O}, \mathrm{Cd}\left(\mathrm{NO}_{3}\right)_{2} \cdot 2 \mathrm{H}_{2} \mathrm{O}, \mathrm{Cr}\left(\mathrm{NO}_{3}\right)_{3} \cdot 9 \mathrm{H}_{2} \mathrm{O}, \quad \mathrm{Ag}-$ $\mathrm{NO}_{3}, \mathrm{Fe}\left(\mathrm{NO}_{3}\right)_{3} \bullet 9 \mathrm{H}_{2} \mathrm{O}, \mathrm{Mg}\left(\mathrm{NO}_{3}\right)_{2} \cdot 6 \mathrm{H}_{2} \mathrm{O}$, 溶于纯净水制 得.

\section{2 合成}

3.2.12 2 乙氧羰甲氧基- $N, N$-二(乙氧羰甲基)氨基苯 (3)的合成

化合物 3 按照文献[30]提供的方案来合成, 将 $2.75 \mathrm{~g}$ (0.025 mol) 2 -氨基酚和 $11.8 \mathrm{~g}(0.13 \mathrm{~mol})$ 氯乙酸加入三 颈烧瓶中, 加入 $25 \mathrm{~mL}$ 氢氧化钠水溶液 $[7.0 \mathrm{~g}(0.17 \mathrm{~mol})$ $\mathrm{NaOH}$ ], 加热至 $100{ }^{\circ} \mathrm{C}$, 缓慢加入氢氧化钠固体保持 $\mathrm{pH}$ 在 10 左右反应 $1 \mathrm{~h}$. 反应物冷却至室温, 浓缩干燥, 加 入 $80 \mathrm{~mL}$ 无水乙醇和 $10 \mathrm{~mL}$ 浓 $\mathrm{H}_{2} \mathrm{SO}_{4}$, 反应回流 $3 \mathrm{~d}$, 冷 却, 过滤, 将固体溶于乙酸乙酯, 用 $10 \%$ 氢氧化钠溶液 
和饱和食盐水各洗涤 1 次，浓缩有机相并经柱层析纯化 得到 $4.04 \mathrm{~g}$ 化合物 3 , 淡棕色油状物, 产率 $42 \%$. ${ }^{1} \mathrm{H}$ NMR $\left(\mathrm{CDCl}_{3}, 400 \mathrm{MHz}\right) \delta: 6.91 \sim 6.88(\mathrm{~m}, 3 \mathrm{H}), 6.80(\mathrm{~d}$, $J=8.0 \mathrm{~Hz}, 1 \mathrm{H}), 4.65(\mathrm{~s}, 2 \mathrm{H}), 4.25 \sim 4.16(\mathrm{~m}, 10 \mathrm{H}), 1.29 \sim$ $1.22(\mathrm{~m}, 9 \mathrm{H})$.

\section{2 .23 -乙氧羰甲氧基-4- $N, N$-二(乙氧羰甲基)氨基苯} 甲醛(4)的合成

将 $1.47 \mathrm{~g}$ (4.00 mmol)化合物 3 和 $395 \mathrm{mg}$ (0.40 mL, $5.00 \mathrm{mmol}$ )吡啶加入 $3.76 \mathrm{~g} \mathrm{DMF}(4.0 \mathrm{~mL}, 50.0 \mathrm{mmol})$ 中, 在 $0{ }^{\circ} \mathrm{C}$ 条件下逐滴加入 $4.94 \mathrm{~g} \mathrm{POCl}_{3}(3.00 \mathrm{~mL}, 32.0$ $\mathrm{mmol}$ ), 5 $10 \mathrm{~min}$ 内滴加完毕. 加热至 $60{ }^{\circ} \mathrm{C}$ 反应 $2 \mathrm{~h}$, TLC 监测反应进程. 反应完毕, 将混合物倾入冰水中, 碳酸钠溶液调节 $\mathrm{pH}$ 至中性, 乙酸乙酯萃取, 有机相以 饱和食盐水洗涤, 无水硫酸镁干燥, 浓缩后柱层析纯化 得到 $0.98 \mathrm{~g}$ 化合物 4, 黄色固体, 产率 62\%. m.p. 54.6 $55.3{ }^{\circ} \mathrm{C} ;{ }^{1} \mathrm{H} \mathrm{NMR}\left(\mathrm{CDCl}_{3}, 400 \mathrm{MHz}\right) \delta$ : 9.77 (s, 1H), 7.41 $(\mathrm{d}, J=8.0 \mathrm{~Hz}, 1 \mathrm{H}), 7.27(\mathrm{~s}, 1 \mathrm{H}), 6.81(\mathrm{~d}, J=8.0 \mathrm{~Hz}, 1 \mathrm{H})$, $4.66(\mathrm{~s}, 2 \mathrm{H}), 4.28(\mathrm{~s}, 4 \mathrm{H}), 4.27 \sim 4.20(\mathrm{~m}, 6 \mathrm{H}), 1.31 \sim 1.27$ (m, 9H); MALDI-TOF MS $m / z: 396.16\left(\mathrm{M}^{+}+1\right)$.

3.2 .33 -乙氧羰甲氧基-4- $N, N-$ 二(乙氧羰甲基)氨基苯 基罗丹明(5)的合成

将 $500 \mathrm{mg}$ (1.26 mmol)化合物 4, $520 \mathrm{mg}$ (3.15 mmol) 3-(二乙基氨基)苯酚和 $36 \mathrm{mg}(0.10 \mathrm{mmol})$ 对甲苯磺酸加 入烧瓶中, 加 $15 \mathrm{~mL}$ 丙酸溶解, 加热至 $65{ }^{\circ} \mathrm{C}$ 反应过夜. 反应完毕, 将反应液冷至室温, 转移至烧杯中加入 100 $\mathrm{mL} 3 \mathrm{~mol} / \mathrm{L} \mathrm{NaOAc}, \mathrm{CHCl}_{3}$ 萃取 $(100 \mathrm{~mL} \times 3)$, 有机相用 无水硫酸钠干燥, 旋蒸除去溶剂, 得深紫色固体. 将深 紫色固体溶于 $20 \mathrm{~mL} \mathrm{MeOH}$ 和 $20 \mathrm{~mL} \mathrm{CH}_{2} \mathrm{Cl}_{2}$, 加入 310 $\mathrm{mg}$ 四氯苯醌(1.26 mmol), 常温搅拌 $2 \mathrm{~h}$, 浓缩后柱层析 纯化 3 次得 $0.22 \mathrm{~g}$ 化合物 $\mathbf{5}$, 深紫色固体, 产率 $25 \%$. m.p. $138.4 \sim 140.2{ }^{\circ} \mathrm{C} ;{ }^{1} \mathrm{H}$ NMR $\left(\mathrm{CDCl}_{3}, 400 \mathrm{MHz}\right) \delta$ : 7.46 (d, $J=12 \mathrm{~Hz}, 2 \mathrm{H}), 6.99$ (d, $J=8.0 \mathrm{~Hz}, 2 \mathrm{H}), 6.90$ (d, $J=8.0 \mathrm{~Hz}, 2 \mathrm{H}), 6.86 \sim 6.83(\mathrm{~m}, 3 \mathrm{H}), 4.67(\mathrm{~s}, 2 \mathrm{H}), 4.31(\mathrm{~s}$, $4 \mathrm{H}), 4.25$ (q, $J=8.0 \mathrm{~Hz}, 6 \mathrm{H}), 3.66$ (q, $J=6.7 \mathrm{~Hz}, 8 \mathrm{H})$, $1.36 \sim 1.30(\mathrm{~m}, 21 \mathrm{H}) ;{ }^{13} \mathrm{C} \mathrm{NMR}\left(\mathrm{CDCl}_{3}, 100 \mathrm{MHz}\right) \delta$ : $165.3,162.5,152.2,151.1,149.6,143.2,136.1,126.5$, 118.9, 118.6, 113.0, 110.4, 108.2, 107.4, 90.8, 60.4, 55.8, $55.4,48.2,40.5,8.6,8.5,7.0$; MALDI-TOF MS $\mathrm{m} / \mathrm{z}$ : $688.36\left(\mathrm{M}^{+}\right)$. Anal. calcd for $\mathrm{C}_{39} \mathrm{H}_{50} \mathrm{~N}_{3} \mathrm{O}_{8}: \mathrm{C} 67.84, \mathrm{H} 7.36$, $\mathrm{N} 6.02$; found $\mathrm{C} 68.00, \mathrm{H} 7.32, \mathrm{~N} 6.10$.

3.2 .43 -着基-4-氨基酚- $N, N, O$-三乙酸铯盐罗丹明 (R1) 的合成

在单颈烧瓶中加入 $40 \mathrm{mg}(0.06 \mathrm{mmol})$ 化合物 $\mathbf{5}, 6$ $\mathrm{mL}$ 甲醇与水 $\left(V_{\text {甲醇 }}: V_{\text {水 }}=1: 1\right)$ 混合作溶剂, 将 $17 \mathrm{mg}$ $(0.03 \mathrm{mmol}) \mathrm{CsOH} \cdot \mathrm{H}_{2} \mathrm{O}$ 加入到上述溶液中, 氩气保护下
加热回流 $16 \mathrm{~h}$. TLC 监测反应进程，反应完全后冷却至 室温, 加入 $10 \mathrm{~mL}$ 水稀释, $\mathrm{CHCl}_{3}(20 \mathrm{~mL} \times 5)$ 洗涤出混 合液中未反应的原料, 收集水相, 真空浓缩干燥得到 55 $\mathrm{mg}$ 产物 R1, 红棕色固体, 产率 $93 \%$. m.p.>300 ${ }^{\circ} \mathrm{C} ;{ }^{1} \mathrm{H}$ NMR $\left(\mathrm{D}_{2} \mathrm{O}, 400 \mathrm{MHz}\right) \delta: 7.41(\mathrm{~d}, J=8.0 \mathrm{~Hz}, 2 \mathrm{H}), 7.23$ (s, $1 \mathrm{H}), 7.13(\mathrm{~d}, J=8.0 \mathrm{~Hz}, 2 \mathrm{H}), 6.96(\mathrm{~s}, 1 \mathrm{H}), 6.75(\mathrm{~s}, 1 \mathrm{H})$, $6.22(\mathrm{~s}, 1 \mathrm{H}), 6.01(\mathrm{~s}, 1 \mathrm{H}), 4.47(\mathrm{~s}, 2 \mathrm{H}), 3.90(\mathrm{~s}, 4 \mathrm{H}), 3.32$ $(\mathrm{q}, J=8.0 \mathrm{~Hz}, 8 \mathrm{H}), 1.25 \sim 1.17(\mathrm{~m}, 12 \mathrm{H})$; MALDI-TOF MS $m / z$ : $999.95\left(\mathrm{M}^{+}\right)$.

\section{3 荧光性能测试}

将化合物 R1 用纯净水配成 $1 \times 10^{-4} \mathrm{~mol} / \mathrm{L}$ 溶液，金 属盐用纯净水配成 $1 \times 10^{-4} \mathrm{~mol} / \mathrm{L}$ 溶液. 取化合物 $\mathbf{R} 1$ 溶 液 $1 \mathrm{~mL}$ 置于 $10 \mathrm{~mL}$ 比色管中, 加入 $1 \mathrm{~mL} 0.01 \mathrm{~mol} / \mathrm{L}$ Tris- $\mathrm{HCl}$ 缓冲溶液 $(\mathrm{pH}=7.2), 1 \mathrm{~mL} 0.1 \mathrm{~mol} / \mathrm{L} \mathrm{KCl}$, 然 后分别加入不同金属离子溶液，用纯净水稀释至刻度. 振荡使之混合均匀后超声 $5 \mathrm{~min}$, 测定其苂光发射光谱 及 $\mathrm{Ca}^{2+}$ 浓度 $(0.0,2.0,4.0,8.0,15.0,30.0,50.0,80.0,100$, $150,200,400,600 \mu \mathrm{mol} / \mathrm{L}$ )对探针分子苂光光谱的影响. $\mathrm{pH}$ 由 $1 \mathrm{~mol} / \mathrm{L} \mathrm{HCl}$ 和 $\mathrm{NaOH}$ 调节.

测试条件: 室温, 样品池为 $1 \mathrm{~cm} \times 1 \mathrm{~cm} \times 4 \mathrm{~cm}$ 石英 比色血, 狭缝宽度 $5 \mathrm{~nm}$, 灵敏度为 2 , 激发波长 $\lambda_{\mathrm{ex}}$ 为 $520 \mathrm{~nm}$, 于 $540 \sim 650 \mathrm{~nm}$ 范围内检测荧光.

辅助材料(Supporting Information) 化合物 3, 4, 5 和探 针 $\mathbf{R} 1$ 的核磁谱图. 这些材料可以免费从本刊网站 (http://sioc-journal.cn/)上下载.

\section{References}

[1] Clapham, D. E. Cell 2007, 131, 1047.

[2] Floto, R. A.; Mahautsmith, M. P.; Somasundaram, B. Cell Calcium $1995,18,377$.

[3] Wojda, U.; Salinska, E.; Kuznicki, J. IUBMB Life 2008, 60, 575.

[4] Pani, B.; Singh, B. B. Cell Mol. Life Sci. 2008, 65, 205.

[5] Keisuke, Y.; Hiroki, A.; Kazuhiko, Y. Opt. Rev. 2005, 12, 415.

[6] Kettlewell, S.; Cabrero, P.; Nicklin, S. A.; T. Dowa, J. A. J. Mol. Cell. Cardiol. 2009, 46, 891.

[7] Harkins, A. B.; Kurebayashi, N.; Baylor, S. M. Biophys. J. 1993, 65,865 .

[8] Gee, K. R.; Archer, E. A.; Lapham, L. A.; Leonard, M. E.; Zhou, Z.-L.; John, B.; Diwu, Z. Bioorg. Med. Chem. Lett. 2000, 10, 1515.

[9] Akwasi, M.; Joseph, P. Y. K.; Roger, Y. T. J. Biol. Chem. 1989, 264,8171 .

[10] Liu, Z.-H.; Li, W.- H.; Shen, P.; Cai, R.-X. Acta Chim. Sinica 2004, 62, 445 (in Chinese).

(刘志洪，李文化，沈萍，蔡汝秀，化学学报, 2004, 62, 445.)

[11] Pieter, A. O.; Robert, E.; Louis, A. L. Bioconjugate Chem. 2001, 12,203

[12] Grzegorz, G.; Martin, P.; Roger, Y. T. J. Biol. Chem. 1989, 260, 3440.

[13] Roger, Y. T. Biochemistry 1980, 19, 2396.

[14] Pieter, A. O.; Robert, E. L.; Louis, A. L. Bioconjugate Chem. 2001, 


\section{2,76 .}

[15] Emmanuel, R.; Fotini, L.; Artemissia, P. N. Cell Calcium 2006, 39, 3.

[16] Boens, N.; Nesibe, A.; Subhendu, S. S.; Amuri, K.; Georges, J. H. Tetrahedron 2006, 62, 684.

[17] Mako, K.; Kai, J. Anal. Chem. 2010, 82, 6472.

[18] Nikola, B.; Mukulesh, B.; Qin, W.-W.; Bert, M.; Mario, S.; Wim, D.; Noel, B. Org. Biomol. Chem. 2005, 3, 2755.

[19] Yu, C.-W.; Zhang, J.; Li, J.-H.; Liu, P. Microchim. Acta 2011, 174, 247.

[20] Tang, L.-J.; Li, F.-F.; Liu, M.-H.; Raju, N. Spectrochim. Acta, Part A 2011, 78, 1168 .

[21] Duong, T. Q.; Jong, S. K. Chem. Rev. 2010, 110, 6280.

[22] Li, J.-B.; Hu, Q.-H.; Zeng, Y.; Yu, X.-L.; Pan, Z.-Q. Prog. Chem. 2012, 24, 823 (in Chinese).

(黎俊波, 胡启辉, 曾阳, 余响林, 潘志权, 化学进展, 2012, 24, 823.)

[23] Zhou, Y.; Wang, F.; Kim, Y.; Kim, S. J.; Yoon, J. Org. Lett. 2009, 11,4442 .
[24] Li, N.; Liu, M.-L.; Yin, W.-T.; Yang, Z.; Li, J.-L.; Shi, Z. Chin. J. Org. Chem. 2011, 31, 39 (in Chinese).

(李娜, 刘美玲, 尹文婷, 杨征, 李剑利, 史真, 有机化学, 2011, 31, 39.)

[25] Du, J.- J.; Fan, J.-L.; Peng, X.- J.; Sun, P. P.; Wang, J.-Y. Org. Lett. 2010, $12,476$.

[26] Sun, W.; Hu, D.-Y.; Wu, Z.-B.; Song, B.-A.; Yang, S. Chin. J. Org. Chem. 2011, 31, 997 (in Chinese).

(孙伟, 胡德禹, 吴志兵, 宋宝安, 杨松, 有机化学, 2011, 31, 997.)

[27] Huang, W.-J.; Wu, W.-H.; Liang, J.-X. Acta Chim. Sinica 2012, 70, 873 (in Chinese).

(黄文君, 吴文辉, 梁嘉香, 化学学报, 2012, 70, 873.)

[28] Yutaka, H.; Rivon, S. K. Theory \& Manufacture Dye Chemistry, Gihodo, K. K., Tokyo, 1963, p. 373, p. 788.

[29] Lin, W.-Y.; Yuan, L.; Cao, Z.-M.; Feng, Y.-M.; Song, J.-Z. Angew. Chem. Int. Ed. 2010, 49, 375 .

[30] Bert, M.; Mario, S.; Noel, B.; Wim, D. Synthesis 2005, 1838.

(Li, L.; Fan, Y.) 


\title{
辅助材料(Supporting Information)
}

\section{新型罗丹明类 $\mathrm{Ca}^{2+}$ 荧光探针的合成及光谱性能研究}

\author{
苏文崎童 杰杨秉勤*
}

(西北大学化学与材料科学学院 合成与天然功能分子化学教育部重点实验室 西安 710069)

\section{Table of Contents}

1. 化合物 3 的 ${ }^{1} \mathrm{H} \mathrm{NMR}$ (图 S1)

2. 化合物 4 的 ${ }^{1} \mathrm{H} N \mathrm{NMR}$ (图 S2)

3. 化合物 $\mathbf{5}$ 的 ${ }^{1} \mathrm{H} \mathrm{NMR}$ (图 S3)

4. 化合物 $\mathbf{5}$ 的 ${ }^{13} \mathrm{C} N \mathrm{NMR}$ (图 S4)

5. 探针化合物 $\mathbf{R} 1$ 的的 ${ }^{1} \mathrm{H}$ NMR（图 S5)

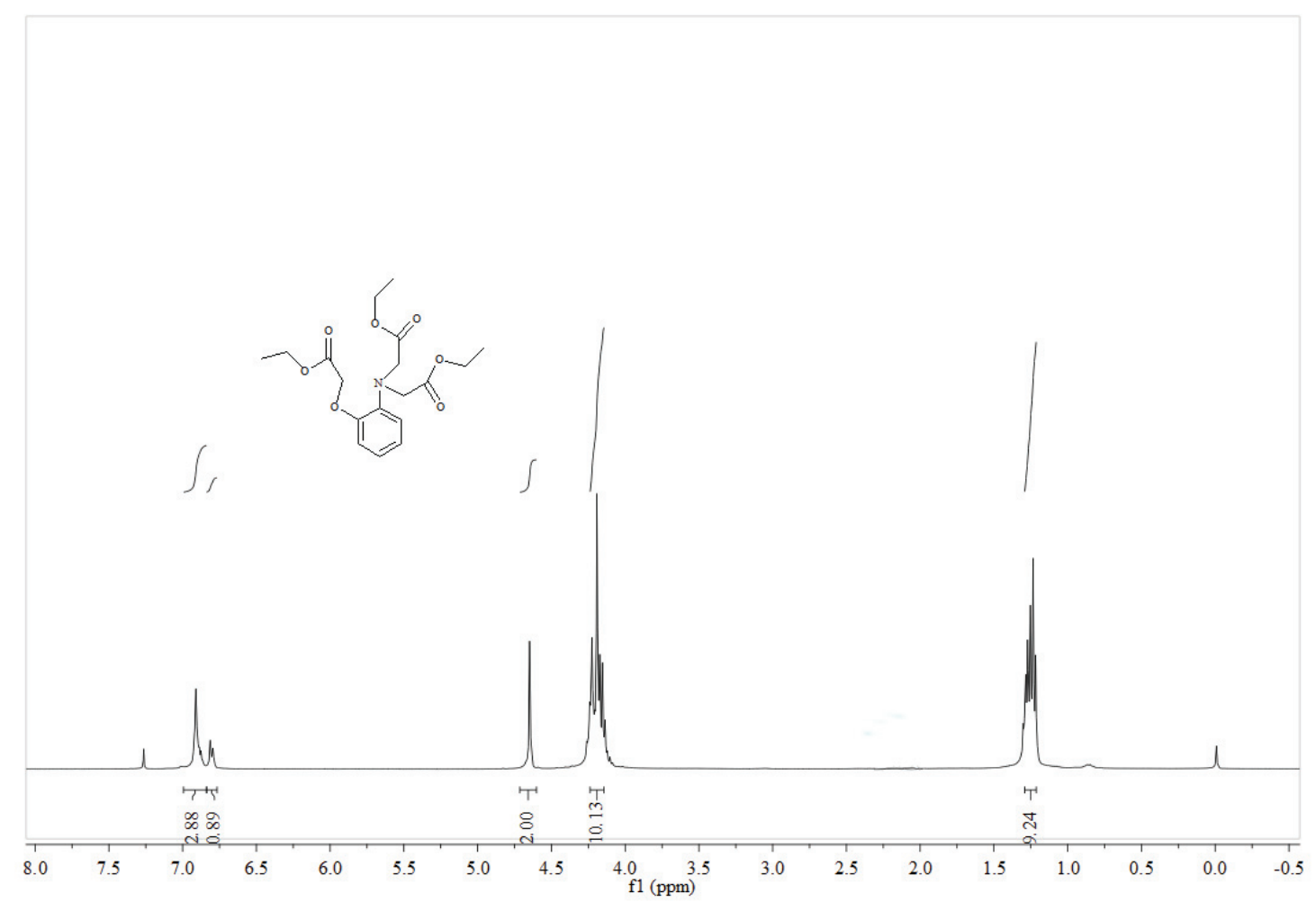

图 S1 化合物 $\mathbf{3}$ 的 ${ }^{1} \mathrm{H}$ NMR

Figure S1 ${ }^{1}$ H NMR spectra of compound 3 

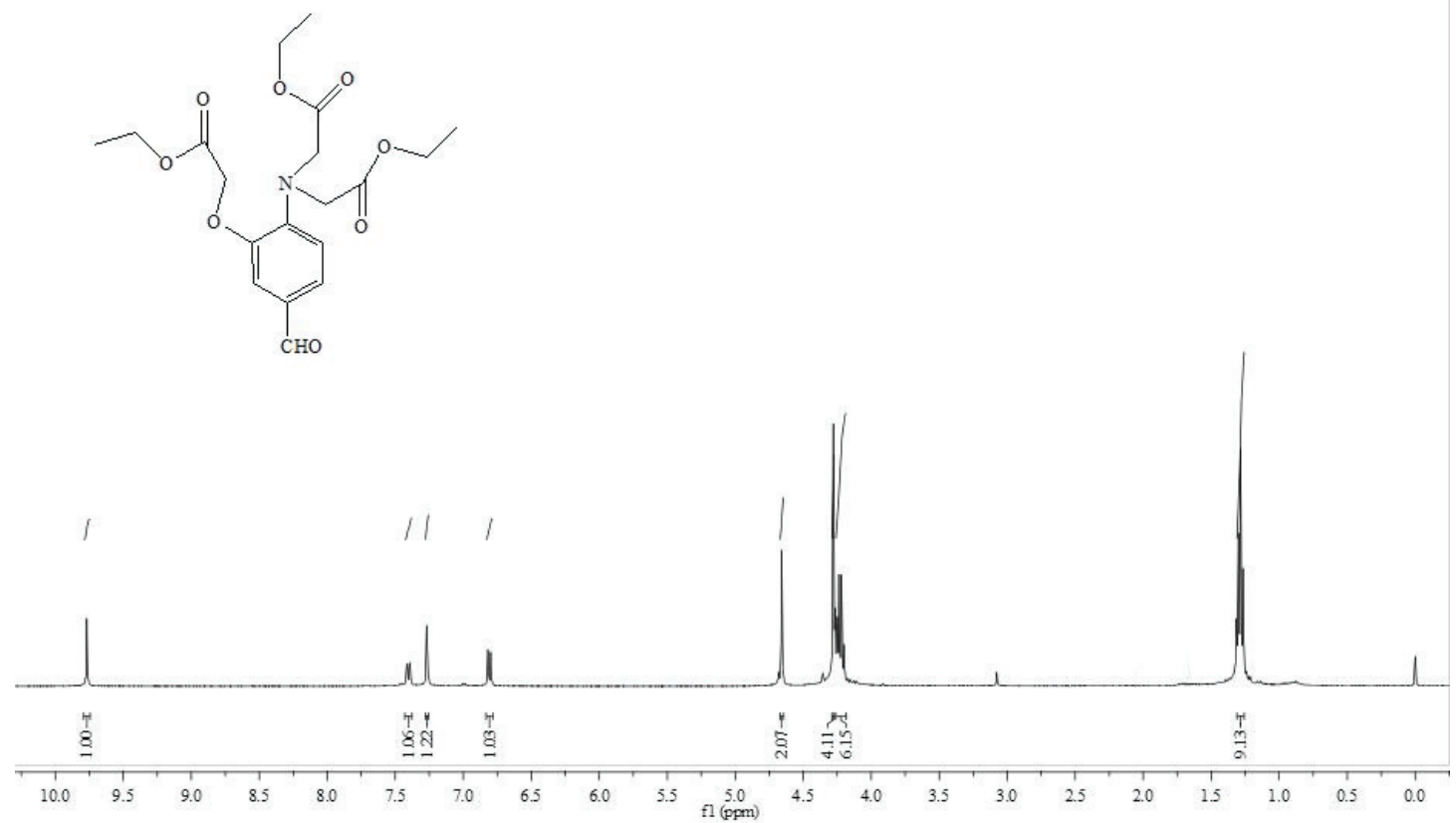

图 S2 化合物 $\mathbf{4}$ 的 ${ }^{1} \mathrm{H}$ NMR

Figure S2 ${ }^{1} \mathrm{H}$ NMR spectra of compound 4

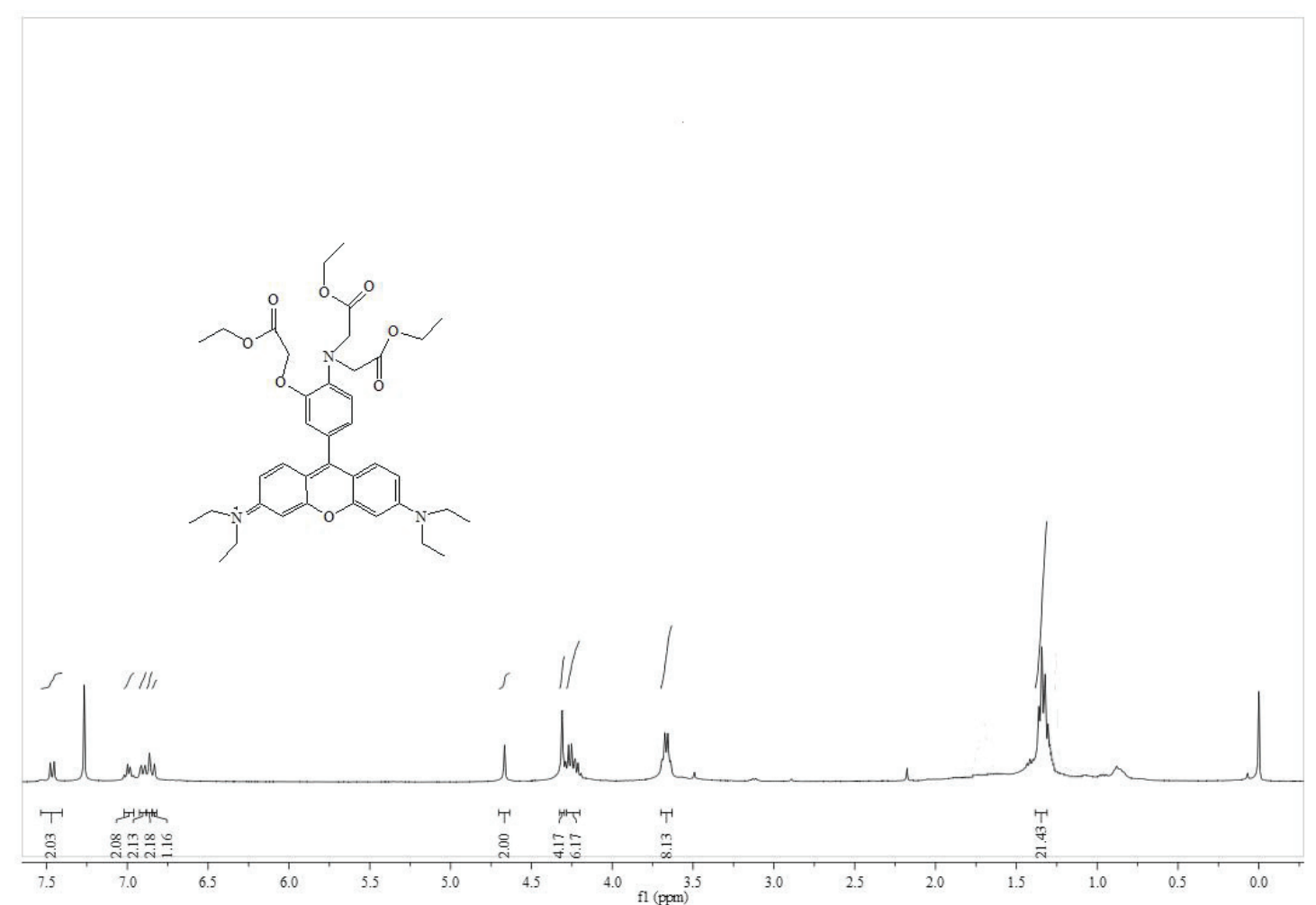

图 S3 化合物 $\mathbf{5}$ 的 ${ }^{1} \mathrm{H}$ NMR

Figure S3 ${ }^{1} \mathrm{H}$ NMR spectra of compound 5 


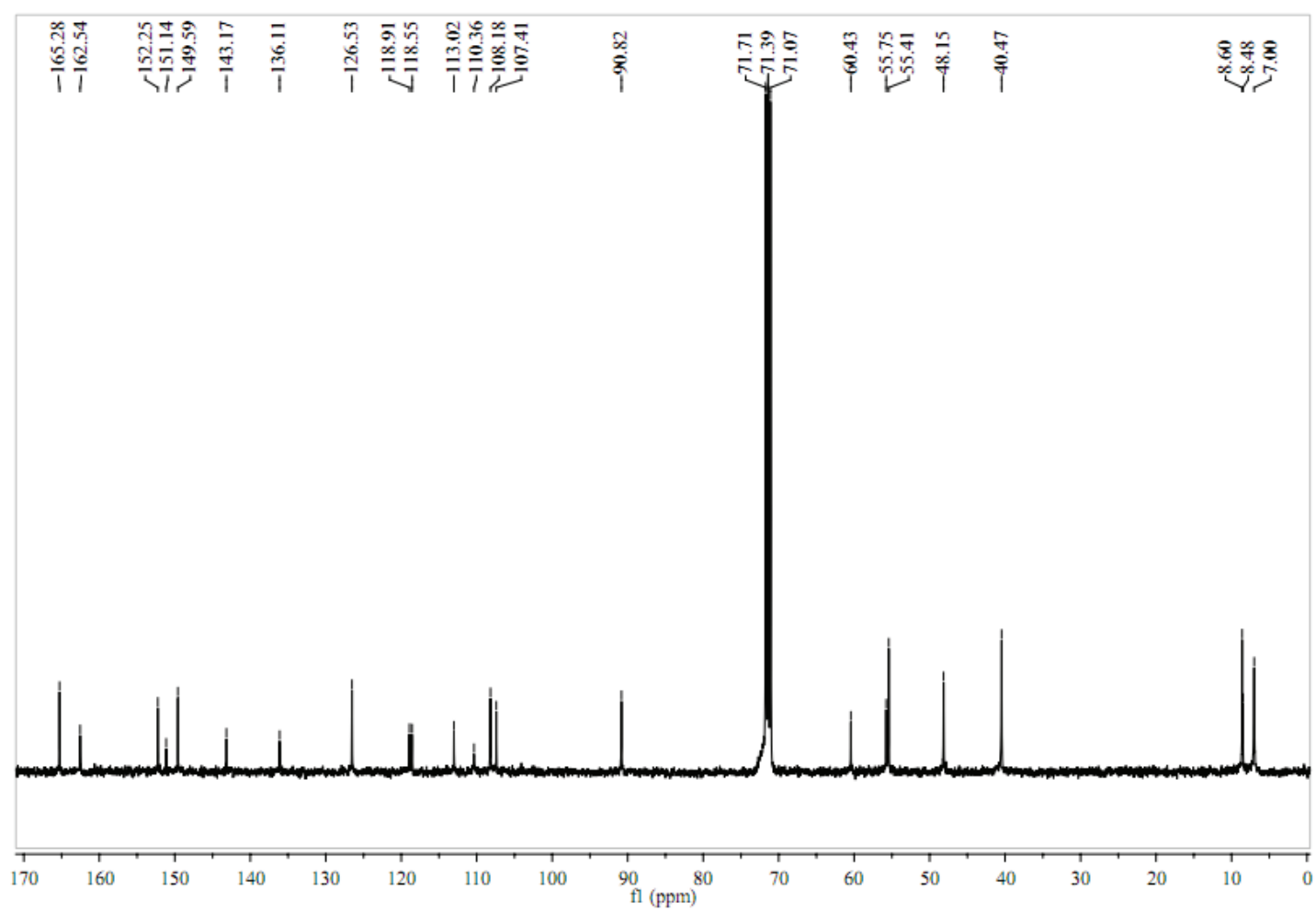

图 S4 化合物 $\mathbf{5}$ 的 ${ }^{13} \mathrm{C} \mathrm{NMR}$

Figure S4 ${ }^{13} \mathrm{C}$ NMR spectra of compound 5

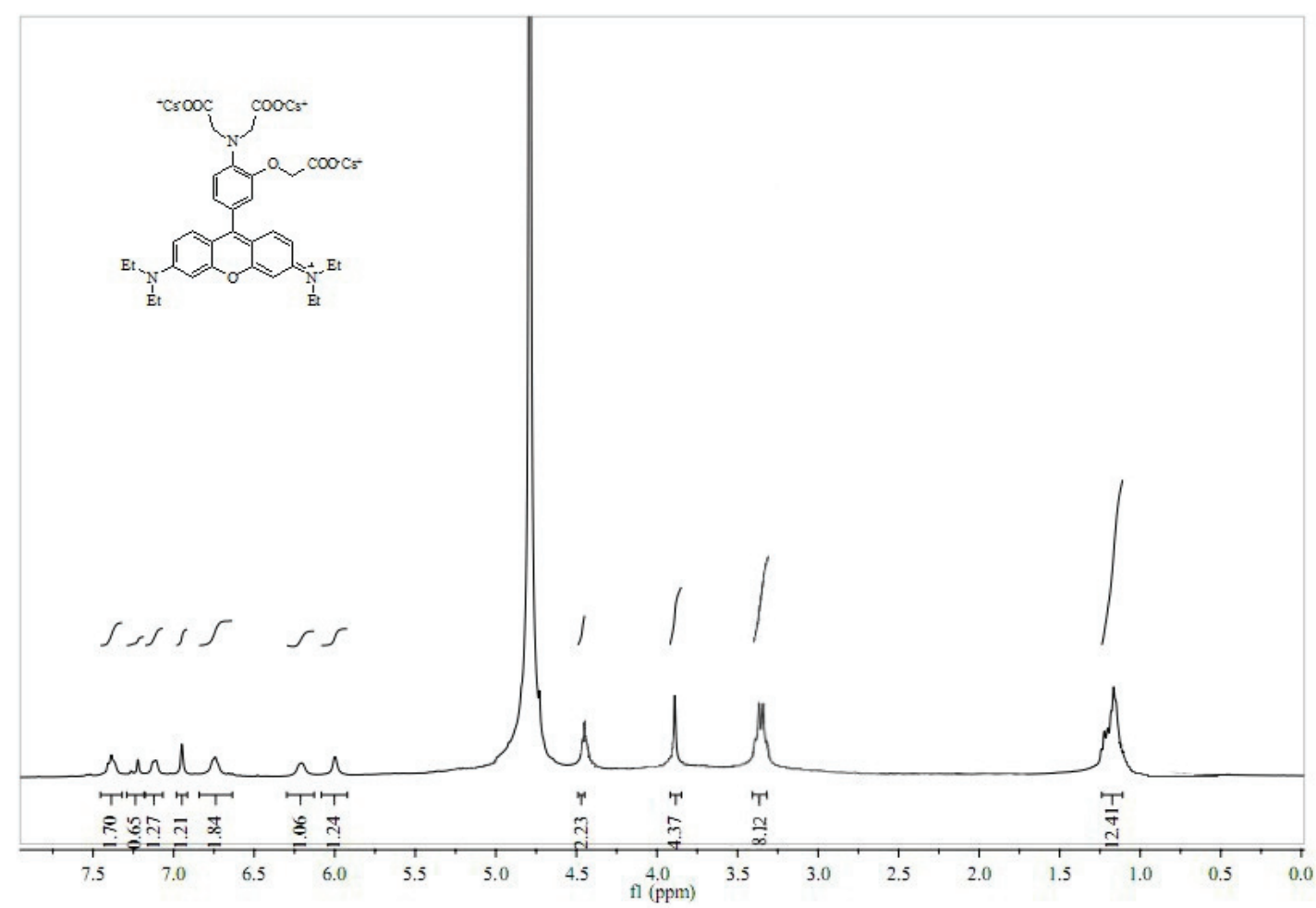

图 S5 探针化合物 R1 的 ${ }^{1} \mathrm{H}$ NMR

Figure S5 $\quad{ }^{1}$ H NMR spectra of compound R1 